\title{
Publisher's Note: Critical and Griffiths-McCoy singularities in quantum Ising spin glasses on $d$-dimensional hypercubic lattices: A series expansion study [Phys. Rev. E 96, 022139 (2017)]
}

\author{
R. R. P. Singh and A. P. Young
}

(Received 5 October 2017; published 11 October 2017)

DOI: 10.1103/PhysRevE.96.049901

This paper was published online on 17 August 2017 with errors in Table I, Fig. 2(a), and related text. The paper has been corrected as of 6 October 2017. The paper is incorrect in the printed version of the journal. For the benefit of the print readership, Fig. 2(a) is replicated below.

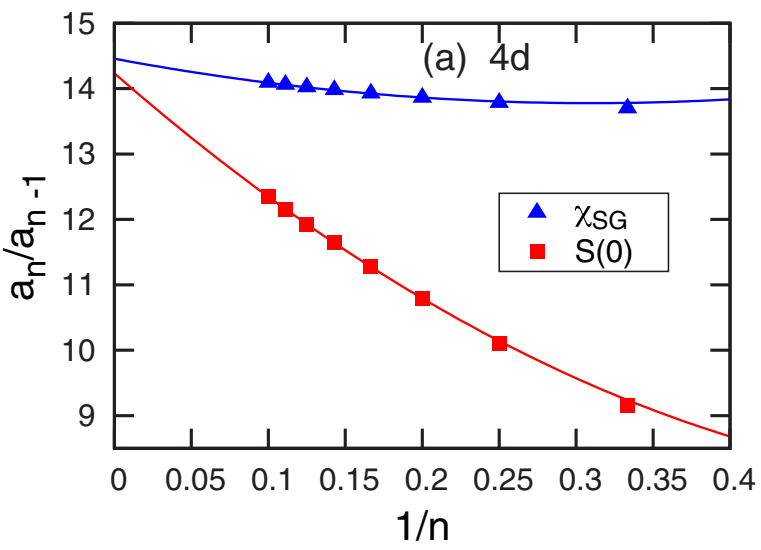

FIG. 2. Ratio plots for (a) $d=4$, (b) $d=3$, and (c) $d=2$. In all cases there is a difference in the intercept, i.e., the value of $1 / x_{c}$, between results for $S(0)$ and results for $\chi_{\mathrm{SG}}$, indicating that $\chi_{\mathrm{SG}}$ diverges in the paramagnetic phase before the QCP is reached, i.e., the singularity in $\chi_{\mathrm{SG}}$ corresponds to GM singularities, not critical singularities. Note that the difference in $x_{c}$ values is very large in two dimensions but rapidly decreases with increasing dimension. The fits are linear for $d=2$ and quadratic for $d=3$ and 4 . Parameters of the fits are listed in Table I. 